\title{
A Data Mining-Based Response Model for Target Selection in Direct Marketing
}

\author{
Eniafe Festus Ayetiran \\ Department of Computer \& Information Systems, Achievers University, Owo, Nigeria \\ Email:eniafef@yahoo.com \\ Adesesan Barnabas Adeyemo \\ University of Ibadan, Ibadan, Nigeria \\ Email: sesan_adeyemo@yahoo.com
}

\begin{abstract}
Identifying customers who are more likely to respond to new product offers is an important issue in direct marketing. In direct marketing, data mining has been used extensively to identify potential customers for a new product (target selection). Using historical purchase data, a predictive response model with data mining techniques was developed to predict a probability that a customer in Ebedi Microfinance bank will respond to a promotion or an offer. To achieve this purpose, a predictive response model using customers' historical purchase data was built with data mining techniques. The data were stored in a data warehouse to serve as management decision support system. The response model was built from customers' historic purchases and demographic dataset.
\end{abstract}

Bayesian algorithm precisely Naïve Bayes algorithm was employed in constructing the classifier system. Both filter and wrapper feature selection techniques were employed in determining inputs to the model.

The results obtained shows that Ebedi Microfinance bank can plan effective marketing of their products and services by obtaining a guiding report on the status of their customers which will go a long way in assisting management in saving significant amount of money that could have been spent on wasteful promotional campaigns.

Index Terms - Data warehouse, Data Mining, Direct Marketing, Target Selection, Naïve Bayes

\section{Introduction}

Large amounts of data are nowadays available to companies about their customers. This data can be used to establish and maintain direct relationship with the customers in order to target them individually for specific products and services offer from the company. Large databases of customers and market data are maintained for this purpose. The customers to be targeted are selected from the database given different types of information such as demographic information and information on the customers' personal characteristics like profession, age and sex and purchase history. Usually, the selected customers are contacted directly through one or some of the following means: personal contact, mail, e-mail, telephone and short message service (sms) to promote the new product or service. This type of marketing is called direct marketing. Among other companies, a growing number of banks and insurance companies are adopting direct marketing as their main strategy for interacting with their customers. Partly due to the growing interest in direct marketing, it has become an important application for data mining. In direct marketing, data mining has been used extensively to identify potential customers for new products. Using historical purchase data, a predictive response model was developed using a data mining technique to predict a probability that a customer is going to respond to a promotion or an offer.

In general, banks and financial services companies in Nigeria use mass marketing as their strategy for promoting a new service or product to their customers. In this strategy, a single communication message is broadcast to all customers through media such as print, radio or television etc. In this approach, companies do not establish a direct relationship with their customers for new product offers. This kind of sales promotion leads to a high waste as only a small proportion of customers respond to these offers. This is largely due to the fact that differences among customers are not put into consideration. As a result, in today's world where products and services are overwhelming and there is a highly competitive market, mass marketing has become less effective. 
Consequent upon the ineffectiveness of mass marketing strategy, banks, financial services companies and other companies are shifting away from this strategy and are now targeting subsets of their customer base for specific product and service offers (Direct marketing) ${ }^{[19][21]}$.In direct marketing, companies and organisations try to establish and maintain direct relationship with their customers in order to target them individually for specific product or service offers. Direct marketing is done by sending product offers and information directly to customers through personal contact, sending e-mails or SMS to customers, making phone calls or by addressing customers through post. Nowadays, this type of marketing is being used by growing number of companies as their main strategy for interacting with their customers. But direct marketers in a wide range of industries from banking, manufacturing among others are faced with the challenge of continually rising printing and postage costs, high telephone, internet and transport costs together with high investment cost on new product and services with decreasing response rates from customers, hence the need to target the likely respondents to their products.

Identifying customers who are likely to respond to new offers will be a difficult task through manual perusal of a large customer database. Hence, data mining technique was employed for pattern recognition in customers' dataset in order to make accurate customers' response prediction.

\subsection{Data Mining}

Data Mining or Knowledge Discovery in Databases can be defined as an activity that extracts some new nontrivial information contained in large databases. The goal is to discover hidden patterns, unexpected trends or other subtle relationships in the data using a combination of techniques from machine learning, statistics and database technologies. This new discipline today finds application in a wide and diverse range of business, scientific and engineering scenarios.

The overall knowledge discovery process was outlined by ${ }^{[22]}$ as an interactive and iterative process involving more or less the following steps: understanding the application domain, selecting the data, data cleaning and preprocessing, data integration, data reduction and transformation, selecting data mining algorithms, data mining, interpretation of the results and using the discovered knowledge. According to ${ }^{[13]}$, data mining tasks can be generally classified into two categories: descriptive and predictive. The former characterizes the general properties of the data in the database. The latter performs inference on the current data in order to make predictions.

Actually, the major reason why data mining has attracted a great deal of attention in the information industry and in the society as a whole in recent years is due to the wide availability of huge amounts of data and the imminent need for turning such data into useful information and knowledge ${ }^{[13]}$. The data mining process is sometimes referred to as knowledge discovery or KDD (knowledge discovery in databases). The term "KDD" (Knowledge Discovery in Databases) refers to the overall process of discovering useful knowledge from data. There is a difference in understanding the terms "knowledge discovery" and "data mining" between people from different areas contributing to this new field.

Knowledge discovery in databases is the process of identifying valid, novel, potentially useful, and ultimately understandable patterns/models in data. Data mining is a step in the knowledge discovery process consisting of particular data mining algorithms that under some acceptable computational efficiency limitations, finds patterns or models in data ${ }^{[14]}$.

\subsection{Bayesian Belief Networks and Naive Bayes Classifier}

Classification is the task of identifying the class labels for instances based on a set of features (attributes). Learning accurate classifiers from pre-classified data is a very active research topic in machine learning and data mining. In the past two decades, many algorithms have been developed for learning which include decision-trees and neural-network classifiers. While Bayesian networks (BNs) are powerful tools for knowledge representation and inference under conditions of uncertainty, they were not considered as classifiers until the discovery that Naïve-Bayes, a very simple kind of Bayesian Networks that assumes the attributes are independent, given the class node, are surprisingly effective in classification $\operatorname{task}^{[6]}$.

A Bayesian network, belief network or directed acyclic graphical model is a probabilistic graphical model that represents a set of random variables and their conditional dependences via a directed acyclic graph (DAG). For example, a Bayesian network could represent the probabilistic relationships between diseases and symptoms. Given symptoms, the network can be used to compute the probabilities of the presence of various diseases. Formally, Bayesian networks are directed acyclic graphs whose nodes represent random variables in the Bayesian sense: they may be observable quantities, latent variables, unknown parameters or hypotheses. Edges represent conditional dependencies. Nodes which are not connected represent variables which are conditionally independent of each other. Each node is associated with a probability function that takes as input a particular set of values for the node's parent variables and gives the probability of the variable represented by the node. For example, if the parents are $m$ Boolean variables, then the probability function could be represented by a table of $2^{m}$ entries, one entry for each of the $2^{m}$ possible combinations of its parents being true or false. Efficient algorithms exist that perform inference and learning in Bayesian networks. Bayesian networks 
that model sequences of variables (e.g. speech signals or protein sequences) are called dynamic Bayesian networks. Generalizations of Bayesian networks that can represent and solve decision problems under uncertainty are called influence diagrams.

The Naive (or simple) Bayes classifer finds its origins in the Bayesian theory of probability. Thomas Bayes (1702-1761) created the Bayes ratio formula, the first definition of conditional probability in the 18th century, but most publications on Bayesian networks and classifiers start to appear between 1980 and 1990 as applications for machine learning (Pearl, 1988). The Bayes Naive classifier selects the most likely classification $V_{n b}$ given the attribute values $x_{1}, x_{2}, \ldots \ldots .$. $\mathrm{x}_{\mathrm{n}}$. This results in:

$V n b=\operatorname{argmaxvj} \epsilon V P(V j) \prod P(x i \mid V j)$

We generally estimate $P\left(a_{i} \mid v_{j}\right)$ using m-estimates:

$$
P(x i \mid v j)=\frac{n c+m p}{n+m}
$$

Where:

$\mathrm{n}=$ the number of training examples for which $\mathrm{v}=\mathrm{v}_{\mathrm{j}}$

$\mathrm{n}_{\mathrm{c}}=$ number of examples for which $\mathrm{v}=\mathrm{v}_{\mathrm{j}}$ and $\mathrm{x}=\mathrm{x}_{\mathrm{i}}$

$\mathrm{p}=$ a priori estimate for $\mathrm{P}\left(\mathrm{x}_{\mathrm{i}} \mid \mathrm{vj}\right)$

$\mathrm{m}=$ the equivalent sample size

A Naïve-Bayes is a simple structure that has the class node as the parent node of all other nodes (Fig 1). No other connections are allowed in a Naïve-Bayes structure. Naïve-Bayes has been used as an effective classifier for many years. Unlike many other classifiers, it is easy to construct, as the structure is given a priori (and hence no structure learning procedure is required). Naïve-Bayes assumes that all the features are independent of each other. Although this independence assumption is obviously problematic, Naïve-Bayes has surprisingly outperformed many sophisticated classifiers over a large number of datasets, especially where the features are not strongly correlated. In recent years, a lot of efforts have focussed on improving Naïve-Bayesian classifiers, following two general approaches: selecting feature subset and relaxing independence assumptions.

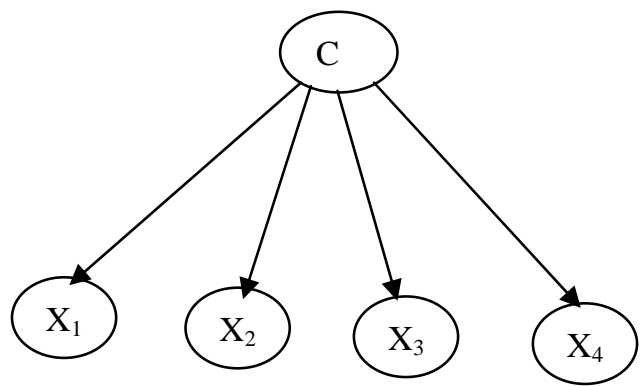

Figure 1: Simple Naive Bayes Classifier

\subsubsection{Advantages of the Naive Bayes classifier}

The main advantage of Bayesian classifiers is that they are probabilistic models, robust to noise found in real data. The Naive Bayes classifier presupposes independence of the attributes used in classification. However, it was tested on several artificial and real datasets, showing good performances even when strong attribute dependences are present. In addition, the Naive Bayes classifier can outperform other powerful classifiers when the sample size is small.

\section{Materials And Methods}

The purchase behaviour variables used in the model development are as follow.

Recency: This is the number of months since the last purchase and first purchase. It is typically the most powerful of the three characteristics for predicting response to a subsequent offer. This seems quite logical. It says that if you have recently purchased something from a company, you are more likely to make another purchase than someone who did not recently make a purchase.

Frequency: This is the number of purchases. It can be the total of purchases within a specific time frame or include all purchases. This characteristic is second to recency in predictive power for response. Again, it is quite intuitive as to why it relates to future purchases.

Monetary value: This is the total naira amount. Similar to frequency, it can be within a specific time frame or include all purchases. Of the three, this characteristic is the least powerful when it comes to predicting response. But when used in combination, it can add another dimension of understanding.

Demographic information includes customers' personal characteristics and information such as age, sex, address, profession etc

\subsection{Data Warehouse Development}

The data used for this research was gathered from customers' records at Ebedi Microfinance Limited. Management staff of the bank were asked questions concerning their products, services and operations which enhanced the understanding of the data gathered. Oracle database version 10.2.0.3 was used in building the data warehouse.The following procedures were adopted in the design of the data warehouse:

\subsubsection{Warehouse Builder Design Centre Preparation}

In this phase, the project is specified. Connection to target data object was established by specifying the locations. In this case, a single data source was used and 
therefore a single oracle module was used ${ }^{[1][10[12][16]}$. The logical design of the warehouse was carried out to support two physical environments: testing and production environments.

\subsubsection{Importing the Source Metadata}

The metadata was imported from a single location. Customers' purchases and demographic data were imported from a text file.

\subsubsection{Profiling Data and Ensuring Data Quality}

Quality data is crucial to decision-making and planning. The aim of building a data warehouse is to have an integrated, single source of data that can be used to make business decisions. Since the data is usually sourced from a number of disparate systems, it is important to ensure that the data is standardised and cleansed before loading into the data warehouse. Data profiling is the process of uncovering data anomalies, inconsistencies and redundancies by analysing the content, structure, and relationships within the data. The analysis and data discovery techniques form the basis for data monitoring.

\subsubsection{Designing the Target Schema}

Relational target schema was designed and used as the target schema. A relational target schema is one that contains relational data objects such as tables, views, materialized views, and sequences. All the warehouse data are stored in these objects. Relational objects which include tables, views, materialized views, and sequences were created. Additional structures pertaining to relational objects such as constraints, indexes and partitions were also created. The objects were configured by setting the physical properties. Finally the codes that will create the data objects were generated.

\subsubsection{Designing the ETL Logic}

ETL (Extraction, Transformation and Loading) logic deals with designing mappings that define the flow of data from the source to target objects. After mapping design, a process flow is defined. A process flow allows activities to be linked together and describes constraints between the activities.

\subsubsection{Deploying the Design and Executing the Data Integration Solution}

Deployment is the process of copying the relevant metadata and generated code in the design centre to the target schema. This step is necessary because it enables the target schema to execute ETL logic such as mappings. Execution is the process of executing the ETL logic defined in the deployed objects.

\subsubsection{Monitoring and Reporting on the Data Warehouse}

It is essential to ensure the quality of data entering your data warehouse over time. Data auditors enable the monitoring of the quality of incoming data by validating incoming data against a set of data rules and determining if the data conforms to the business rules defined in the data warehouse.

\subsection{Data Mining and Response Model Development}

The dataset derived from the data warehouse for the mining activity had 14 attributes including purchases history data and customers' demographic information. The attributes, their data types and description are presented in Table 1.

Table 1: Attributes of Customers Purchases History and Demographic dataset.

\begin{tabular}{|c|c|c|}
\hline Attribute & Data Type & Description \\
\hline ACC_NO & Numerical & $\begin{array}{l}\text { A unique number } \\
\text { identifying each customer }\end{array}$ \\
\hline SURNAME & Categorical & A customer's family name \\
\hline FIRST_NAME & Categorical & A customer's given name \\
\hline CONTACT_MODE & Categorical & $\begin{array}{l}\text { Media through which a } \\
\text { customer is contacted }\end{array}$ \\
\hline PROFESSION & Categorical & Customer's occupation \\
\hline SEX & Categorical & Customer's gender \\
\hline DATEOFBIR & Categorical & Customer's date of birth \\
\hline MARISTAT & Categorical & $\begin{array}{ll}\text { Customer's marital } \\
\text { information }\end{array}$ \\
\hline ADDRESS & Categorical & Customer's contact address \\
\hline ACCOUNT TYPE & Categorical & $\begin{array}{l}\text { Account type operated by a } \\
\text { customer }\end{array}$ \\
\hline OPEN DATE & Categorical & $\begin{array}{l}\text { Date on which an account } \\
\text { was opened }\end{array}$ \\
\hline AMOUNT(N) & Numerical & $\begin{array}{l}\text { Opening amount for an } \\
\text { account }\end{array}$ \\
\hline DATLASTTR & Categorical & $\begin{array}{l}\text { Date on last transaction was } \\
\text { carried out }\end{array}$ \\
\hline TNT & Numerical & $\begin{array}{l}\text { Total number of transactions } \\
\text { by a customer }\end{array}$ \\
\hline
\end{tabular}

Methods for analysing and modelling data can be divided into two groups: supervised learning and unsupervised learning. Supervised learning requires input data that has both predictor (independent) variables and a target (dependent) variable whose value is to be estimated. Through various means, the process learns how to model (predict) the value of the target variable based on predictor variables. Decision trees, neural networks, support vector machines are examples of supervised learning. Supervised learning is best suited for analysis dealing with the prediction of some variable.

Unsupervised learning on the other hand, instead of identifying a target (dependent) variable treats all of the 
variables equally. In this case, the goal is not to predict the value of a variable but rather to look for patterns, groupings or other ways to characterise the data which may lead to understanding of the way data interrelates. Cluster analysis, correlation and statistical measures are examples of unsupervised learning.

The purpose of predictive mining is to find useful patterns in the data in order to make nontrivial predictions on new data. Two major categories of predictive mining techniques are those which express the mined results as a black box whose innards are effectively incomprehensible to non-experts and those which represent the mined results as a transparent box whose construction reveals the structure of the pattern.

Neural networks are major techniques in the former category. The latter includes methods for constructing decision trees, classification rules, bayesian network, association rules, clusters and instance-based learning [5][7][9].

In this work, the Naive Bayesian algorithm (a supervised learning algorithm) was used to model the customers' purchases dataset for target selection whose construction are easily comprehensible to experts and non-experts alike. Figure 2 presents a pictorial representation of the classifier system phases. Sixty percent $(60 \%)$ of the dataset were used to build the response model and the model was applied to the remaining forty percent (40\%) of the dataset for testing.

\subsubsection{Feature Selection}

Feature selection is a critical step in response modelling. No matter how powerful a model is, presence of irrelevant input variables lead to poor accuracy. Because customer related dataset usually contains hundreds of features or variables, many of which are irrelevant and heavily correlated with others, without feature selection, they tend to deteriorate performance of the model, as well as increase the model training time. Feature subset selection can be formulated as an optimization problem which involves searching the space of possible features to identify a subset that is optimum or near optimal with respect to performance measures such as accuracy. Feature selection can then either be performed as a preprocessing step, independent of the induction algorithm or explicitly make use of it. The former approach is termed filter, the latter wrapper. Filter methods operate independently of the target learning algorithm. Undesirable inputs are filtered out of the data before induction commences. Wrapper methods make use of the actual target learning algorithm to evaluate the usefulness of inputs. Typically, the input evaluation heuristic that is used is based upon inspection of the trained parameters and/or comparison of predictive performance under different input subset configurations. Input selection is then often performed in a sequential fashion. The backward selection scheme starts from a full input set and step-wisely prunes input variables that are undesirable. The forward selection scheme starts from the empty input set and step-wisely adds input variables that are desirable. Feature wrappers often achieve better results than filters due to the fact that they are tuned to the specific interaction between an induction algorithm and its training data, but it also tends to be more computationally expensive than the filter model.

When the number of features becomes very large, the filter model is usually chosen due to its computational efficiency. In this study, both filter and wrapper methods have been employed. Attributes such as surname, first name and mode of contact have been removed from the input as part of pre-processing due to their irrelevance in the prediction activity. However, attributes such as sex, date of birth, profession, date of last transaction, amount involved in the account opening etc have been used as inputs and left for the induction algorithm to determine their importance in the prediction task with the prediction value as the target.

\section{Results And Discussion}

\subsection{Pattern Recognition}

This is the stage where patterns representing knowledge in the mined results are identified and evaluated based on some evaluation metrics.

\section{Evaluation Metrics}

In selecting the appropriate algorithm and features that best model customers' response, the following evaluation metrics were employed:

i. Percentage of Correct/Incorrect Classification: This is the difference between the actual and predicted values of variables.

ii. True Negative (TN): Number of correct predictions that an instance is false.

iii. False Positive (FP): Number of incorrect predictions that an instance is true

iv. False Negative (FN): Number of incorrect predictions that an instance is false.

v. True Positive (TP): Number of correct predictions that an instance is true.

vi. Accuracy (Acc): Proportion of total number of predictions that were correct.

$$
\text { Accuracy }=\frac{T P+T N}{T P+F P+T N+F N}
$$

vii. Error Rate (E): Proportion of total number of predictions that were incorrect

Error Rate $=1-$ Acc 
viii. Confusion Matrix: The most basic performance measures can be described by a confusion matrix. The confusion matrix (Kohavi, 1988) contains information about actual and predicted classifications done by a classification system. The columns represent the predicted classifications, and the rows represent the actual (true) classifications for each of all records.

ix. Receiver Operating Characteristics (ROC) Analysis: The Receiver Operating Characteristic (ROC) analysis comes from statistical decision theory (Green and Swets, 1966) and was originally used during World War II for the analysis of radar images. It is a graphical plot of the sensitivity of True Positive Rates (TPR) to False Positive Rate (TFR) varying the discrimination threshold.

\subsection{Performance Metrics}

The accuracy (Acc) and Error rate (E) are widely used metrics for measuring the performance of learning systems. However, when the prior probabilities of the classes are very different, such metric might be misleading. Accuracy alone is not an adequate measure of performance especially where the number of negative cases is much greater than the number of positive cases.

Lift/Gain Chart: In such cases where the number of negative cases is much greater than the number of positive cases, a lift chart can also be used. The lift chart (also called Gain chart) gives a graphical representation of these parameters for various thresholds on the output and encapsulates all the information contained in the confusion matrix.

Table 2 presents some samples of the Naïve Bayes Prediction results obtained.

Table 2: Sample Naïve Bayes Prediction Results

\begin{tabular}{|c|c|c|c|c|c|}
\hline Acc_No & $\begin{array}{l}\text { Predic } \\
\text { tion }\end{array}$ & Remark & Probability & Cost & 丞 \\
\hline 700002 & 1 & Respondent & 1 & 1.70E-08 & 1 \\
\hline 200003 & 1 & Respondent & 0.9998502 & 3.31E-04 & 1 \\
\hline 300004 & 1 & Respondent & 0.9999341 & $1.46 \mathrm{E}-04$ & 1 \\
\hline 200005 & 0 & $\begin{array}{c}\text { Non } \\
\text { Respondent }\end{array}$ & 0.72576463 & 0.50051033 & 1 \\
\hline 500006 & 1 & Respondent & 1 & $6.89 \mathrm{E}-16$ & 1 \\
\hline 100007 & 0 & $\begin{array}{c}\text { Non } \\
\text { Respondent }\end{array}$ & 0.8347949 & 0.30151784 & 1 \\
\hline 100008 & 0 & $\begin{array}{c}\text { Non } \\
\text { Respondent }\end{array}$ & 0.8267257 & 0.316245 & 1 \\
\hline 100009 & 1 & Respondent & 1 & $1.17 \mathrm{E}-12$ & 1 \\
\hline 100010 & 1 & Respondent & 0.9999999 & $2.74 \mathrm{E}-07$ & 1 \\
\hline
\end{tabular}

\begin{tabular}{|c|c|c|c|c|c|}
\hline 200011 & 1 & Respondent & 1 & $1.29 \mathrm{E}-16$ & 1 \\
\hline 300012 & 1 & Respondent & 1 & $4.84 \mathrm{E}-17$ & 1 \\
\hline 200013 & 1 & Respondent & 1 & $1.57 \mathrm{E}-12$ & 1 \\
\hline 200014 & 1 & Respondent & 0.99943364 & 0.00125279 & 1 \\
\hline 100015 & 0 & $\begin{array}{c}\text { Non } \\
\text { Respondent }\end{array}$ & 1 & $4.89 \mathrm{E}-09$ & 1 \\
\hline 100016 & 0 & $\begin{array}{c}\text { Non } \\
\text { Respondent }\end{array}$ & 0.48024932 & 0.9486032 & 1 \\
\hline 100017 & 1 & Respondent & 1 & $1.20 \mathrm{E}-17$ & 1 \\
\hline 600018 & 1 & Respondent & 0.99988407 & $2.56 \mathrm{E}-04$ & 1 \\
\hline 100019 & 1 & Respondent & 0.99930745 & 0.00153182 & 1 \\
\hline 100020 & 1 & Respondent & 0.99996334 & $8.11 \mathrm{E}-05$ & 1 \\
\hline 500021 & 1 & Respondent & 1 & $1.02 \mathrm{E}-16$ & 1 \\
\hline 100022 & 1 & Respondent & 0.9998686 & $2.91 \mathrm{E}-04$ & 1 \\
\hline
\end{tabular}

\subsection{Summary of Prediction Report}

Table 3 gives a summary of the response prediction results obtained by the Naïve Bayes classifier when applied to the customers' dataset.

Table 3: Summary of Response Prediction Report

\begin{tabular}{|c|c|}
\hline Response & Number of Customers \\
\hline Respondents & 221 \\
\hline Non Respondents & 179 \\
\hline Total & 400 \\
\hline
\end{tabular}

Percentage Positive Response $=221 / 400 * 100$

$$
=55.25 \%
$$

Percentage Negative Response $=179 / 400 * 100$

$$
=44.75 \%
$$

From the summary of results, the level of likely favorable response of customers is slightly above average because the percentage of likely positive response is slightly above $50 \%$ (55.25\%) while that of likely unfavorable response is slightly below average because the percentage of negative response is slightly below $50 \%$ (44.75\%). The implication of this is that Ebedi Microfinance bank will benefit from sending promotion offers to the potential respondents and save cost as a result of not sending promotion offers to the non respondents. This will consequently increase the bank's Return on Investment.

Naïve Bayes Response Model Evaluation and Performance

The summary results of response model evaluation are presented in Table 4 . Table 5 presents the statistics of the model performance the confusion matrix is presented in Table 6 
Table 4: Results of Response Model Evaluation

\begin{tabular}{|c|c|}
\hline Metrics & Value \\
\hline True Positive Rate & 0.7654320987 \\
\hline False Positive Rate & 0.4594594594 \\
\hline Average Accuracy & 0.6529863197 \\
\hline Overall Accuracy & 0.6580645161 \\
\hline Error Rate(1 -Accuracy) & 0.3419354839 \\
\hline Cost & 53 \\
\hline Probability Threshold & 0.3787237704 \\
\hline
\end{tabular}

Table 5: Model Performance Statistics

\begin{tabular}{|r|c|c|c|c|}
\hline Target & $\begin{array}{c}\text { Total } \\
\text { Actual }\end{array}$ & $\begin{array}{l}\text { Correctly } \\
\text { Predicted (\%) }\end{array}$ & Cost & $\begin{array}{c}\text { Cost } \\
\text { (\%) }\end{array}$ \\
\hline $\mathbf{0}$ & 74 & 59.46 & 66.36 & 58.32 \\
\hline $\mathbf{1}$ & 81 & 67.9 & 47.45 & 41.69 \\
\hline
\end{tabular}

Table 6: Confusion Matrix

\begin{tabular}{|l|l|l|l|l|l|}
\hline & 0 & 1 & Total & Correct \% & Cost \\
\hline 0 & $44(\mathrm{TN})$ & $30(\mathrm{FP})$ & 74 & 59.46 & 66.36 \\
\hline 1 & $26(\mathrm{FN})$ & $55(\mathrm{TP})$ & 81 & 67.9 & 47.45 \\
\hline Total & 70 & 85 & 155 & & \\
\hline Correct & 62.86 & 64.71 & & & \\
\hline Cost & 47.45 & 66.36 & & & \\
\hline
\end{tabular}

From the confusion matrix the difference between the correctness produced by the actual classification (represented by the row interpretation) and the predicted classification (represented by the column interpretation) for the positive cases (in which we are interested) shows a higher correctness (67.9\%) for the actual value which suggests the accuracy of the model.

From the confusion matrix the difference between the correctness produced by the actual classification (represented by the row interpretation) and the predicted classification (represented by the column interpretation) for the positive cases (in which we are interested) shows a higher correctness (67.9\%) for the actual value which suggests the accuracy of the model.

\subsubsection{Receiver Operating Characteristics (ROC) Curve}

Figure 2 presents the plot of True Positive Rates (TPR) against the False Positive Rates (FPR) with the value of the TPR being 0.7654320987 (the intersection of the ROC curve and the probability threshold) and the value of FPR as 0.4594594594 (the intersection of the diagonal and the probability threshold). From the ROC result presented in Table 7, it can be observed that the model produces higher True Positive (TP) and False positive (FP) cases as the probability threshold increases. The True Positive Rate (TPR) and the False Positive Rate (FPR) produces 0.7654320987 and 0.4594594594 respectively. This implies the model produces a higher number of correctly identified respondents as against a lower number of non respondents incorrectly identified as respondents. This attests to the model's accuracy.

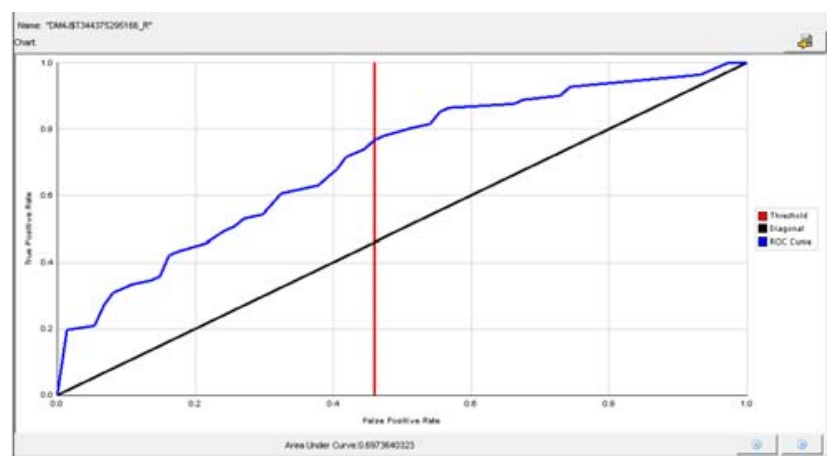

Figure 2: Receiver Operating Characteristics (ROC) Curve

\subsubsection{Lift Charts}

The cumulative Lift chart is presented in Figure 3. The cumulative quantile for first quantile is 1.7939814815. The cumulative positive for first quantile is 0.1851851852 . The cumulative lift for the other quantiles up to the tenth quantile together with cumulative positive, threshold, target density count, percentage record cumulative, target cumulative and non target cumulative is presented in Table 8

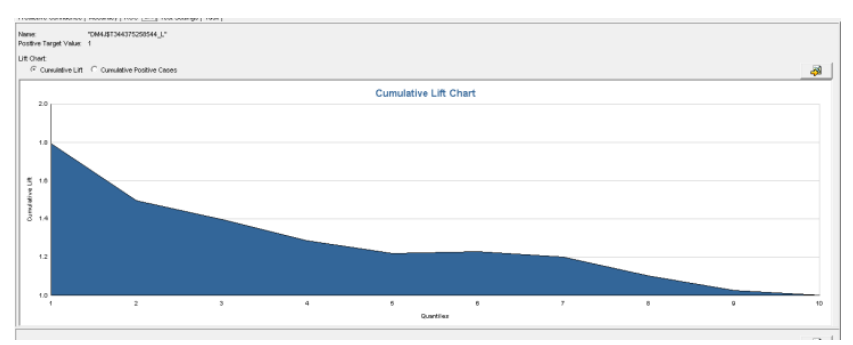

Figure 3: Cumulative Lift chart

Looking at the steeply increasing cumulative lift chart, the model was able to achieve a high degree of positive cases (1.8 out of 2.0 for 10 quantiles), that is, high number of correctly identified respondents.

The Lift result presented in Table 8 shows that the model achieves a good number of targets (respondents) out of the total count (total number of customers addressed) on the first six quantiles, sending promotion offers to more customers after the sixth quantile will not justify the cost of the promotion campaign because only a few targets are gotten from the total count. 
Table 7: Receiver Operating Characteristics (ROC) Result

\begin{tabular}{|c|c|c|c|c|c|c|c|c|}
\hline Index & $\begin{array}{c}\text { Probability } \\
\text { Threshold }\end{array}$ & $\begin{array}{c}\text { False } \\
\text { Positive }\end{array}$ & $\begin{array}{c}\text { False } \\
\text { Negative }\end{array}$ & $\begin{array}{c}\text { True } \\
\text { Positive }\end{array}$ & $\begin{array}{c}\text { True } \\
\text { Negative }\end{array}$ & Accuracy & $\begin{array}{l}\text { Average } \\
\text { Accuracy }\end{array}$ & Cost \\
\hline 0 & 1 & 0 & 81 & 0 & 74 & 0.477419355 & 0.5 & 81 \\
\hline 1 & 1 & 1 & 65 & 16 & 73 & 0.574193548 & 0.592008675 & 66 \\
\hline 2 & 0.99999994 & 4 & 64 & 17 & 70 & 0.561290323 & 0.577911245 & 68 \\
\hline 3 & 0.999988914 & 5 & 59 & 22 & 69 & 0.587096774 & 0.602018685 & 64 \\
\hline 4 & 0.999983847 & 6 & 56 & 25 & 68 & 0.6 & 0.613780447 & 62 \\
\hline 5 & 0.999983072 & 7 & 55 & 26 & 67 & 0.6 & 0.61319653 & 62 \\
\hline 6 & 0.9999578 & 8 & 54 & 27 & 66 & 0.6 & 0.612612613 & 62 \\
\hline 7 & 0.999951899 & 10 & 53 & 28 & 64 & 0.593548387 & 0.605271939 & 63 \\
\hline 8 & 0.999943256 & 11 & 52 & 29 & 63 & 0.593548387 & 0.604688021 & 63 \\
\hline 9 & 0.999915421 & 12 & 47 & 34 & 62 & 0.619354839 & 0.628795462 & 59 \\
\hline 10 & 0.999897599 & 13 & 46 & 35 & 61 & 0.619354839 & 0.628211545 & 59 \\
\hline 11 & 0.999818563 & 16 & 44 & 37 & 58 & 0.612903226 & 0.620286954 & 60 \\
\hline 12 & 0.999719083 & 18 & 41 & 40 & 56 & 0.619354839 & 0.625291959 & 59 \\
\hline 13 & 0.999652863 & 19 & 40 & 41 & 55 & 0.619354839 & 0.624708041 & 59 \\
\hline 14 & 0.9995296 & 20 & 38 & 43 & 54 & 0.625806452 & 0.630296964 & 58 \\
\hline 15 & 0.999390244 & 22 & 37 & 44 & 52 & 0.619354839 & 0.62295629 & 59 \\
\hline 16 & 0.771490574 & 24 & 32 & 49 & 50 & 0.638709677 & 0.640306974 & 56 \\
\hline 17 & 0.680298388 & 28 & 30 & 51 & 46 & 0.625806452 & 0.625625626 & 58 \\
\hline 18 & 0.581525683 & 29 & 28 & 53 & 45 & 0.632258065 & 0.631214548 & 57 \\
\hline 19 & 0.549383104 & 30 & 26 & 55 & 44 & 0.638709677 & 0.63680347 & 56 \\
\hline 20 & 0.460064292 & 31 & 23 & 58 & 43 & 0.651612903 & 0.648565232 & 54 \\
\hline 21 & 0.408490717 & 33 & 21 & 60 & 41 & 0.651612903 & 0.647397397 & 54 \\
\hline 22 & 0.37872377 & 34 & 19 & 62 & 40 & 0.658064516 & 0.65298632 & 53 \\
\hline 23 & 0.356039256 & 35 & 18 & 63 & 39 & 0.658064516 & 0.652402402 & 53 \\
\hline 24 & 0.25514707 & 38 & 16 & 65 & 36 & 0.651612903 & 0.644477811 & 54 \\
\hline 25 & 0.203911513 & 40 & 15 & 66 & 34 & 0.64516129 & 0.637137137 & 55 \\
\hline 26 & 0.154189616 & 41 & 12 & 69 & 33 & 0.658064516 & 0.648898899 & 53 \\
\hline 27 & 0.001631624 & 42 & 11 & 70 & 32 & 0.658064516 & 0.648314982 & 53 \\
\hline 28 & 2.07E-04 & 49 & 10 & 71 & 25 & 0.619354839 & 0.607190524 & 59 \\
\hline 29 & 1.90E-04 & 50 & 9 & 72 & 24 & 0.619354839 & 0.606606607 & 59 \\
\hline 30 & 8.22E-05 & 54 & 8 & 73 & 20 & 0.6 & 0.585752419 & 62 \\
\hline 31 & 6.90E-05 & 55 & 6 & 75 & 19 & 0.606451613 & 0.591341341 & 61 \\
\hline 32 & $1.74 \mathrm{E}-08$ & 69 & 3 & 78 & 5 & 0.535483871 & 0.515265265 & 72 \\
\hline 33 & $1.52 \mathrm{E}-08$ & 70 & 2 & 79 & 4 & 0.535483871 & 0.514681348 & 72 \\
\hline 34 & 4.10E-12 & 72 & 0 & 81 & 2 & 0.535483871 & 0.513513514 & 72 \\
\hline 35 & 0 & 74 & 0 & 81 & 0 & 0.522580645 & 0.5 & 74 \\
\hline
\end{tabular}


Table 8: Lift/Gain Result

\begin{tabular}{|c|c|c|c|c|c|c|c|c|c|c|c|}
\hline $\begin{array}{l}\text { 营 } \\
\text { 善言 }\end{array}$ & 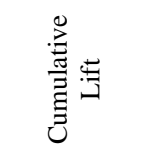 & 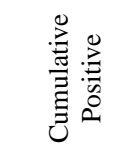 & 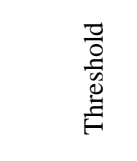 & 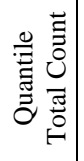 & 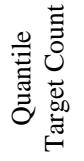 & 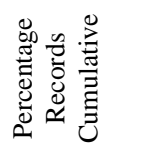 & 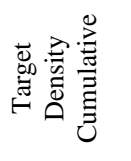 & 䓌莺 & 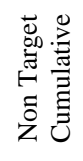 & 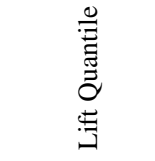 & 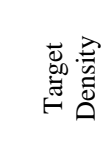 \\
\hline 1 & $\begin{array}{c}1.7939814 \\
81\end{array}$ & $\begin{array}{c}0.185185 \\
185\end{array}$ & 5.39E-08 & 16 & 15 & 0.10322581 & 0.9375 & 15 & 1 & $\begin{array}{c}1.7939814 \\
81\end{array}$ & 0.9375 \\
\hline 2 & $\begin{array}{c}1.5547839 \\
51\end{array}$ & $\begin{array}{c}0.320987 \\
654\end{array}$ & $5.22 \mathrm{E}-05$ & 16 & 11 & 0.20645161 & 0.8125 & 26 & 6 & $\begin{array}{c}1.3155864 \\
2\end{array}$ & 0.6875 \\
\hline 3 & $\begin{array}{c}1.3554526 \\
75\end{array}$ & $\begin{array}{c}0.419753 \\
086\end{array}$ & $2.74 \mathrm{E}-04$ & 16 & 8 & 0.30967742 & $\begin{array}{c}0.708333 \\
3 \\
\end{array}$ & 34 & 14 & $\begin{array}{c}0.9567901 \\
23 \\
\end{array}$ & 0.5 \\
\hline 4 & $\begin{array}{c}1.2557870 \\
37\end{array}$ & $\begin{array}{c}0.518518 \\
519\end{array}$ & $\begin{array}{c}0.001040 \\
4\end{array}$ & 16 & 8 & 0.41290323 & 0.65625 & 42 & 22 & $\begin{array}{c}0.9567901 \\
23\end{array}$ & 0.5 \\
\hline 5 & $\begin{array}{c}1.1959876 \\
54\end{array}$ & $\begin{array}{c}0.617283 \\
951\end{array}$ & $\begin{array}{c}0.932694 \\
2\end{array}$ & 16 & 8 & 0.51612903 & 0.625 & 50 & 30 & $\begin{array}{c}0.9567901 \\
23\end{array}$ & 0.5 \\
\hline 6 & $\begin{array}{c}1.2488628 \\
98 \\
\end{array}$ & $\begin{array}{c}0.765432 \\
099 \\
\end{array}$ & $\begin{array}{c}1.412885 \\
5 \\
\end{array}$ & 15 & 12 & 0.61290323 & $\begin{array}{c}0.652631 \\
6 \\
\end{array}$ & 62 & 33 & $\begin{array}{c}1.5308641 \\
98 \\
\end{array}$ & 0.8 \\
\hline 7 & 1.2003367 & $\begin{array}{c}0.851851 \\
852 \\
\end{array}$ & $\begin{array}{c}1.828681 \\
3 \\
\end{array}$ & 15 & 7 & 0.70967742 & $\begin{array}{c}0.627272 \\
7 \\
\end{array}$ & 69 & 41 & $\begin{array}{c}0.8930041 \\
15 \\
\end{array}$ & $\begin{array}{c}0.4666 \\
6667 \\
\end{array}$ \\
\hline 8 & $\begin{array}{c}1.1175308 \\
64\end{array}$ & $\begin{array}{c}0.901234 \\
568\end{array}$ & $\begin{array}{c}2.211720 \\
5\end{array}$ & 15 & 4 & 0.80645161 & 0.584 & 73 & 52 & $\begin{array}{c}0.5102880 \\
66\end{array}$ & $\begin{array}{c}0.2666 \\
6667\end{array}$ \\
\hline 9 & $\begin{array}{c}1.0388007 \\
05\end{array}$ & $\begin{array}{c}0.938271 \\
605 \\
\end{array}$ & $\begin{array}{c}2.211916 \\
2 \\
\end{array}$ & 15 & 3 & 0.90322581 & $\begin{array}{c}0.542857 \\
1 \\
\end{array}$ & 76 & 64 & $\begin{array}{c}0.3827160 \\
49 \\
\end{array}$ & 0.2 \\
\hline 10 & 1 & 1 & $\begin{array}{c}2.211956 \\
5 \\
\end{array}$ & 15 & 5 & 1 & $\begin{array}{c}0.522580 \\
6 \\
\end{array}$ & 81 & 74 & $\begin{array}{c}0.6378600 \\
82 \\
\end{array}$ & $\begin{array}{c}0.3333 \\
3333 \\
\end{array}$ \\
\hline
\end{tabular}

\section{Conclusion}

From the summary of the prediction results obtained, which classifies $55.25 \%$ of the customers as respondents and $44.75 \%$ as non respondents, we can conclude that Ebedi Microfinance bank can plan effective marketing of its products/services through the guiding report obtained on each of the customers. This will enable the management increase its sales by targeting the respondents and prevent wasteful expenditure that will have been incurred as a result of sending promotion offers to the non respondents. These will go a long way in increasing the bank's Return on Investment.

\section{References}

[1] Adamson C. and M. Venerable. "Data Warehouse Design Solutions”. J. Wiley \& Sons, Inc., 1998

[2] Alejandro Gutiérrez and Adriana Marotta. "An Overview of Data Warehouse Design Approaches and Techniques", 2000

[3] Almquist, E. and Wyner G. "Boost Your Marketing ROI with Experimental Design”. Harvard Business Review, p.135141., 2001

[4] Ballard. C. "Data Modelling Techniques for Data Warehousing”. SG24-2238-00. IBM Red Book. ISBN number 0738402451., 1998

[5] Berry, M. J. A. and Linoff, G. S. "Data Mining Technique for Marketing, Sale, and Customer Relationship Management" (2nd edn), Indiana, Indianapolis Publishing Inc., 2004
[6] Changyun Wang. "Bayesian Belief Network Simulation”, Department of Computer Science Florida State University, 2003

[7] Chen, H.Y., Chen, T.C., Min, D., Fischer, G. and Wu, Y.M.'Prediction of tacrolimus blood levels by using the neural network with genetic algorithm in liver transplantation patients', Therapeutic Drug Monitoring, 21, 1, 50- 56., 1999

[8] Cheung, K.-W., Kwok, J. K., Law, M. H. and Tsui, K.-C. 'Mining customer product rating for personalized marketing ', Decision Support Systems, 35, 231-243., 2003

[9] Chiu, C. "A case-based customer classification approach for direct marketing”, Expert Systems with Applications, 22, 163-168., 2002

[10] Colin McGregor. Oracle Database 2 Day DBA, $10 \mathrm{~g}$ Release 2 (10.2), 2005

[11] Date, C. J. An introduction to database systems (7th ed.). Reading, Mass.: Addison-Wesley, 2000

[12] Gang Luo. Techniques for Operational Data Warehousing. $\mathrm{PhD}$ Thesis (Computer Sciences), University of WiscosinMadison, 2004

[13] Han, J., \& Kamber, M. Data mining: Concepts and Techniques. San Francisco: Morgan Kaufmann Publishers., 2001

[14] Ho, T.B. (nd). Knowledge Discovery and Data Mining Techniques and Practice Available on URL:http://www.netnam.vn/unescocourse/knowlegde/knowfr m.htm,_2006

[15] Inmon. W.H. Building the Data Warehouse. Wiley Computer Publishing, 1996

[16] Scalzo, B. (2003). Oracle DBA guide to data warehousing and star schemas. Upper Saddle River, N.J.: Prentice Hall PTR. [17] Shapiro, G. P. 'Knowledge Discovery in Databases: 10 years after', SIGKDD Explor. Newsl, 1, 2 (Jan. 2000), 59- 61. 
Available from: http://doi.acm.org/10.1145/846183.846197, 2000

[18] Shin, H. J. and Cho, S. 'Response modelling with support vector machines', Expert Systems with Applications, 30, 4, 746-760., 2006

[19] Ou, C., Liu, C., Huang, J. and Zhong, N. 'One Data mining for direct marketing', Springer-Verlag Berlin Heidelberg, pp. 491-498., 2003

[20] Paul Lane, Viv Schupmann and Ingrid Stuart. Oracle Database Data Warehousing Guide 11g Release 1(11.1), 2007

[21] Petrison, L. A., Blattberg, R. C. and Wang, P. 'Database marketing: Past present, and future', Journal of Direct Marketing, 11, 4, 109-125, 1997

[22] Usama M. Fayyad, Gregory Piatetsky-Shapiro, and Padhraic Smyth. From Data Mining to knowledge Discovery: An Overview, Advances in Knowledge Discovery and Data Mining, (1996). AAAI Press, , pp 1-34.

Eniafe Festus Ayetiran is a Lecturer at the Achievers University, Owo, Nigeria. He holds a Master of Science (M.Sc) degree of the University of Ibadan, Nigeria and a Bachelor of Science (B.sc Hons) degree of the Adekunle Ajasin University, Akungba Akoko, Nigeria. His research interests include Data Mining, Data Warehousing, Business Modeling, Software Engineering and Computer Security.

Dr. Adesesan Barnabas Adeyemo is senior lecturer at the Computer Science Department of the University of Ibadan, Nigeria. He obtained his $\mathrm{PhD}$ and M.Sc degrees at the Federal University of Technology, Akure. His research activities are in Data Mining, Data Warehousing \& Computer Networking. He is a member of the Nigerian Computer Society and the Computer Professionals Registration Council of Nigeria. Dr. Adeyemo is a Computer Systems and Network Administration specialist with expertise in Data Analysis and Data Management. 